\title{
Time savings - realized and potential - and fair compensation for community health workers in Kenyan health facilities: a mixed-methods approach
}

\author{
Laura D Sander ${ }^{1 *}$, David Holtzman², Mark Pauly ${ }^{3}$ and Jennifer Cohn ${ }^{4}$
}

\begin{abstract}
Background: Sub-Saharan Africa faces a severe health worker shortage, which community health workers (CHWs) may fill. This study describes tasks shifted from clinicians to CHWs in Kenya, places monetary valuations on CHWs' efforts, and models effects of further task shifting on time demands of clinicians and CHWs.

Methods: Mixed methods were used for this study. Interviews were conducted with 28 CHWs and 19 clinicians in 17 health facilities throughout Kenya focusing on task shifting involving CHWs, time savings for clinicians as a result of task shifting, barriers and enabling factors to $\mathrm{CHWs}^{\prime}$ work, and appropriate CHW compensation. Twenty CHWs completed task diaries over a 14-day period to examine current CHW tasks and the amount of time spent performing them. A modeling exercise was conducted examining a current task-shifting example and another scenario in which additional task shifting to CHWs has occurred.

Results: CHWs worked an average of 5.3 hours per day and spent $36 \%$ of their time performing tasks shifted from clinicians. We estimated a monthly valuation of US\$ 117 per CHW. The modeling exercise demonstrated that further task shifting would reduce the number of clinicians needed while maintaining clinic productivity by significantly increasing the number of CHWs.

Conclusions: CHWs are an important component of healthcare delivery in Kenya. Our monetary estimates of current CHW contributions provide starting points for further discussion, research and planning regarding CHW compensation and programs. Additional task shifting to CHWs may further offload overworked clinicians while maintaining overall productivity.
\end{abstract}

Keywords: task shifting, community health workers, Kenya, compensation

\section{Background}

There is an estimated deficit of 2.4 million doctors, nurses and midwives globally. The majority of this deficit lies in sub-Saharan Africa, which accounts for $24 \%$ of the global burden of disease yet has only $3 \%$ of the world's healthcare workers (HCWs) [1]. Determining how to expand the healthcare workforce and enhance its quality is necessary for affected countries to achieve their health-related millennium development goals (MDGs)

\footnotetext{
* Correspondence: Isande15@jhmi.edu

'Johns Hopkins Bloomberg School of Public Health, 1000 E. Eager Street, Baltimore, MD 21202, USA

Full list of author information is available at the end of the article
}

[1-4]. Task shifting - the reassignment of clinical roles by transferring suitable tasks from higher- to lower-skilled HCWs - is one of the strategies proposed to mitigate the effects of the HCW shortage.

Community health workers (CHWs) are a unique cadre of HCWs and are well suited to help address the HCW gap. Many studies have demonstrated that CHWs can be as effective as more highly skilled HCWs in delivering a variety of services [5-14] and help achieve MDGs [15]. But until recently, there have been few large-scale, sustainable CHW programs implemented in developing countries. Known barriers to successful implementation of $\mathrm{CHW}$ programs include inadequate compensation of 
CHWs, frequent unreimbursed out-of-pocket expenditures and variability in quality and duration of training and supervision [16-20].

Kenya's 2006 Community Strategy (CS) outlines primary health care delivery at the community unit (CU). CUs comprise volunteer CHWs who are linked to the primary health facility through trained Community Health Extension Workers (CHEWs) employed in primary care facilities; each $\mathrm{CHEW}$ is meant to supervise $25 \mathrm{CHWs}$ [21]. CHWs are men and women recruited from the community, 'having demonstrated attitudes valued by the community', and, preferably, are literate [21]. Main tasks performed by CHWs are categorized as: 1) disease prevention and control, 2) family health services, and 3) hygiene and environmental sanitation [22]. Further, the CS stipulates that volunteer CHWs should be paid a stipend 'based on work actually done' [21]. In a 2011 telegram, Kenya's Ministry of Public Health and Sanitation stated 'where funds are available community health workers shall be entitled to a payment of Ksh.2000 per month [approximately USD\$ 25] as performance based incentive' [23]. This statement lacks guidance on where funds should come from and on standardized criteria for performance standards. It remains unclear if and how many CHWs are compensated for their work.

Clinical officers and nurses are also integral to health care at the CU. Clinical officers undergo at least 3 years of university-level training and a year of internship, providing patient care and management at the primary health care level $[24,25]$. Nurses undergo 1 to 4 years of training and, accordingly, achieve certificates, diplomas or degrees [26]. Kenya is facing a severe HCW shortage, and attrition among clinical officers is particularly high at the dispensary level [27,28]. In 2010, the average monthly wage for clinical officers was USD\$ 372, for nurses it was USD\$248, and for public health officers it was USD\$ 124 (J. Mwitari, personal communication, March 2010).

In practice, diverse and distinct community health models have emerged since 2010 when Kenya decentralized health care administration [29]. Faith-based and civil society organizations have played an important role in expanding the government's efforts to establish CUs [30]. As Kenya works toward its One Million Community Health Workers Campaign to standardize CUs, it is important to detail tasks that volunteer CHWs perform. In addition, no studies have looked at the actual time savings realized by task shifting from higher HCW cadres to CHWs [18]. This information is needed to inform implementation of task shifting and help developing country governments, donors and non-governmental organizations (NGOs) better understand the value of CHWs. This mixed-methods study in Kenya was conducted to fill this knowledge gap.

\section{Methods}

\section{Study setting and population}

This was a collaborative, mixed-methods study between the University of Pennsylvania and GROOTS Kenya, a community-based organization that supports CHWs throughout Kenya. Semistructured interviews (qualitative) and task diaries (quantitative) were used to triangulate and develop valuation of CHW tasks; both methods were then used to develop and initiate clinic modeling, the second quantitative component [31].

Seventeen health facilities within the GROOTS network were chosen using quota sampling based on the population densities of the community. Based on demographic data, health facilities were categorized to be in densely, mid- or sparsely populated areas [32,33]. More facilities in sparsely populated areas were selected since the majority of Kenyans reside in rural settings. Facilities included dispensaries, health centers and district hospitals. Site visits occurred between October 2010 and March 2011.

The leader of each health facility was asked to identify 1 to 3 clinical officers and/or nurses and 1 to $3 \mathrm{CHWs}$ for possible enrolment in the study. Potential subjects were approached and informed consent obtained. Study participants were required to be predominately facilitybased and are distinguished from CHEWs, as the latter supervise CHWs and do not directly conduct healthrelated tasks. CHWs are therefore defined as a primary health worker caring for community members. In addition, they are volunteers: monies received for services were largely applied toward procurement of supplies or transportation to outreach community members and also were typically not consistent. Furthermore, monies came from associated NGOs, and not the Ministry of Health. The University of Pennsylvania Institutional Review Board approved the study.

\section{Semistructured interviews}

Semistructured interviews were conducted with all study participants. Clinician interviews focused on 1) assessing tasks shifted from clinicians to $\mathrm{CHWs}$, 2) estimating time freed by task shifting and 3) identifying additional tasks that could be shifted to CHWs. CHW interviews focused on 1) current tasks performed, 2) self-perception of their ability to perform these tasks, 3) views on additional tasks that could be shifted to CHWs and 4) additional support needed to improve performance and permit further task shifting. All participants were asked to estimate an appropriate monthly salary for local CHWs based on their current work. Interviews were recorded, transcribed, and analyzed using Microsoft Excel.

\section{Task diaries}

Task diaries were distributed to CHWs to complete over a consecutive 14-day period. The task diary contained a 
list of common CHW tasks developed by GROOTS Kenya administrators and representative CHWs, and provided space for additional tasks to be entered. Each day CHWs were asked to record every task performed, the number of patients reached with each task, and the amount of time spent per task. Diary data were analyzed using Microsoft Excel.

\section{Analysis of semistructured interviews and task diaries}

Two research staff (LS and DH) conducted and analyzed the semistructured interviews. Tasks recorded in the $\mathrm{CHW}$ diaries were categorized as a directly-shifted or added-value task. Directly-shifted tasks were 'core services' traditionally performed by clinicians (for example, patient registration, measuring vital signs and administering immunizations). All other tasks were considered to be added-value services, which are generally not provided unless a $\mathrm{CHW}$ is available (for example, providing individual and group education and counseling, mobilizing communities). Categorization of tasks was based on consensus of clinicians and CHWs interviewed, and are displayed in Table 1. The total and proportional amount of time spent performing each task was calculated for every $\mathrm{CHW}$ and aggregated by category. Data were analyzed by population density and facility type.

The monetary value of time CHWs spent performing directly-shifted tasks was estimated using wage data for public health officers, clinical officers and nurses obtained by personal interview with the Ministry of Public Health and Sanitation in March 2010 (J. Mwitari, personal communication, March 2010). Since nurses provide most clinical services at dispensaries while clinical officers do so in hospital-linked clinics, nurse salary was used for dispensaries and clinical officer salary for

\section{Table 1 List of clinic services}

\begin{tabular}{ll}
\hline $\begin{array}{l}\text { Core services (services generally } \\
\text { provided) }\end{array}$ & $\begin{array}{l}\text { Added value services (services } \\
\text { generally not provided unless } \\
\text { a CHW is available) }\end{array}$ \\
\hline Patient registration & $\begin{array}{l}\text { Group health education talks } \\
\text { Community mobilization }\end{array}$ \\
Billing & $\begin{array}{l}\text { Social work support } \\
\text { Time with clinician }\end{array}$ \\
Filing & Nutrition support \\
Measuring vital signs* & Defaulter tracing \\
Basic and intensive adherence & Linking health facility and \\
monitoring and counseling* & community \\
Lab services* & \\
Immunizations* & \\
Medication and supply distribution* & \\
HIV voluntary counseling and testing* &
\end{tabular}

*Denotes 'directly-shifted' services designated by clinical officers, nurses and CHWs as tasks traditionally performed by clinicians that could be shifted to CHWs. CHW, community health worker. hospital clinics. An average of clinical officer and nurse salaries was used for health centers. Clinicians and CHWs perceived CHWs to be less efficient than clinicians, likely a result of inadequate $\mathrm{CHW}$ training. Accordingly, the time a $\mathrm{CHW}$ spent performing directly-shifted tasks was reduced by a correction factor of two-thirds, based upon discussions with clinicians and CHWs during the interviews. This adjusted time was multiplied by the salary figure corresponding to the facility type where the $\mathrm{CHW}$ worked to arrive at an estimate of a lower bound monetary value of time each CHW spent on directly-shifted tasks.

Since CHWs are not typically compensated, we had no direct way of valuing the added-value services they provide. Hence, assumptions about the intrinsic value of this work were made. We felt that the work of a $\mathrm{CHW}$ was commensurate with a public health officer, and learned the average monthly wage of that cadre of health workers is USD\$124 per month. This figure is commensurate with the average wage suggested by clinicians in semistructured interviews (see Results), and we felt would be a good starting point by which to value the CHW' added-value services. This wage was multiplied by the time each $\mathrm{CHW}$ spent performing added-value tasks to arrive at a valuation of these services. All analyses were conducted using Microsoft Excel.

\section{Modeling}

A model clinic was created to examine the effects of shifting various tasks from clinicians to CHWs on the time demands of clinicians and CHWs. Figure 1 depicts the progression of patients through a clinic visit and highlights some services patients may require.

The model was created using Microsoft Excel with the following primary inputs: 1) number of patients seen each day at the clinic, 2) number of patients seen each day by one clinician, 3) the proportion of patients requiring a service, and 4) the amount of time to complete each service. Patient volumes were derived from interviews with clinicians. Table 2 outlines all of the inputs for model assumptions, which were based on the authors' clinical experience working in Kenyan health facilities.

A determination was then made for each service based on the information from clinician and $\mathrm{CHW}$ interviews regarding which cadre currently performs the service and which cadre would ideally perform it so as to best utilize health worker skills (Table 2). The main tasks targeted for task shifting are denoted by ${ }^{(*)}$ in Table 1.

The time demands for CHWs and clinicians were then calculated for these 'current' and 'idealized' task allocation scenarios. A sensitivity analysis was performed in which the amount of time required for each cadre to complete a service was varied by a factor of two to determine the effects of these assumptions in the model. 


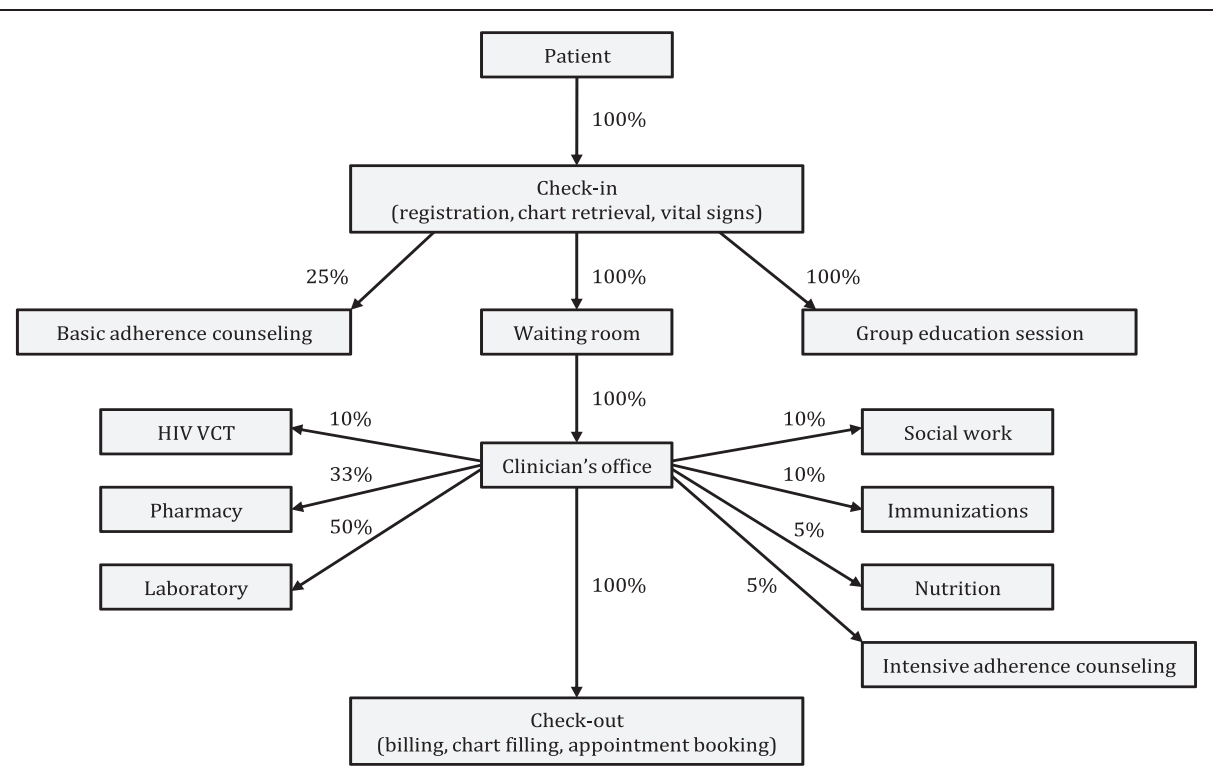

Figure 1 Model clinic flow. A model clinic was created to examine the effects of shifting various tasks from clinicians to community health workers. The figure depicts the progression of patients through a clinic visit and highlights some of the various services they may require. Percentages indicate the estimated proportion of patients requiring that service, based on interviews with clinicians and the authors' clinical experience working in Kenyan health facilities. Table 2 displays all assumptions that were used to create the model clinic.

\section{Results}

Eight locations incorporating 17 health facilities (7 hospitals, 6 health centers and 4 dispensaries) were visited in Nairobi, Nyanza, Central, Eastern and Western Provinces. Ten sites were in sparsely populated areas, three in mid-populated regions and four in densely populated areas.

Forty-seven interviews were conducted with $28 \mathrm{CHWs}$ and 19 clinicians. No potential participant declined to be interviewed. All $28 \mathrm{CHWs}$ interviewed agreed

Table 2 Assumptions used to develop the model clinic

\begin{tabular}{|c|c|c|c|c|}
\hline Task & $\begin{array}{l}\text { Percentage/Number of } \\
\text { patients requiring service }\end{array}$ & $\begin{array}{l}\text { Average time to complete } \\
\text { service per patient (minutes) }\end{array}$ & $\begin{array}{l}\text { Cadre currently } \\
\text { performing task }\end{array}$ & $\begin{array}{l}\text { Cadre ideally } \\
\text { performing task }\end{array}$ \\
\hline Check-in (registration, chart retrieval, vitals) & $100 \%$ & 3 & Clerk & Clerk \\
\hline Vital signs measurement & $100 \%$ & 3 & Clinician & $\mathrm{CHW}$ \\
\hline Clinician visit & $100 \%$ & 7 & Clinician & Clinician \\
\hline Lab services & $50 \%$ & 5 & Clinician & $\mathrm{CHW}$ \\
\hline Medication and supply distribution & $33 \%$ & 5 & Clinician & $\mathrm{CHW}$ \\
\hline Immunizations & $10 \%$ & 5 & Clinician & $\mathrm{CHW}$ \\
\hline Basic adherence counseling & $25 \%$ & 5 & Clinician & $\mathrm{CHW}$ \\
\hline Intensive adherence counseling & $5 \%$ & 10 & Clinician & $\mathrm{CHW}$ \\
\hline Nutrition support & $5 \%$ & 15 & Various & $\mathrm{CHW}$ \\
\hline Social work support & $10 \%$ & 15 & Social work & Social work \\
\hline HIV voluntary counseling and testing & $10 \%$ & 10 & Various & $\mathrm{CHW}$ \\
\hline Check-out (billing, chart filing, appointment booking) & $100 \%$ & 5 & Clerk & Clerk \\
\hline Group education session & $100 \%$ & 30 & $\mathrm{CHW}$ & $\mathrm{CHW}$ \\
\hline Defaulter tracking & 2 & 30 & $\mathrm{CHW}$ & $\mathrm{CHW}$ \\
\hline Community mobilization & - & 60 & $\mathrm{CHW}$ & $\mathrm{CHW}$ \\
\hline Linking facility and community & - & 60 & $\mathrm{CHW}$ & $\mathrm{CHW}$ \\
\hline Patients seen per clinician per day & 25 & - & - & - \\
\hline Patients attending clinic per day & 35 & - & - & - \\
\hline
\end{tabular}


to complete the task diaries; 20 diaries were returned; and 3 were not included in the analysis due to incorrect recording of time spent performing tasks. Overall, the CHWs spent $60 \%$ of their time in facilities and $40 \%$ of their time in the community. Tasks performed in the community include defaulter tracing and linking community members to the health facility. Counseling and health education occurred both in the community and facility; all other tasks occurred in the facility.

\section{Semistructured interviews}

CHWs reported performing a variety of tasks (Table 3). For each of these tasks, clinicians also were asked if these tasks have been shifted from their duties. CHWs and clinicians report varying levels of task shifting. $\mathrm{CHW}$ s provided an important link between health facilities and communities by performing defaulter tracing, home visits and outreach education; interviewees highlighted this link as previously missing or inadequate. The majority of CHWs performed tasks that have been shifted from other cadres, including health education, counseling, taking vital signs, and dressing simple wounds. CHWs and clinicians both cited the need for additional training, reliable provision of supplies, consistent and adequate compensation, and transportation support in order to improve the work of CHWs to enable them to take on more tasks and to retain them.

Clinicians estimated that $\mathrm{CHWs}$ save the clinicians an average of 2.5 hours of work per day (SD 1.1 hours). The average monthly salary estimated by interviewees to be fair compensation for the CHWs' current work was US\$ 160 (SD US\$ 110); CHWs provided a higher estimate than clinicians (US\$ 182 for CHWs, US\$ 128 for clinicians).

\section{Task diaries}

Table 4 presents data from the CHW task diaries on the number of hours CHWs spent performing core services

Table 3 Tasks performed by community health workers (CHWs) and clinician-reported status of task shifting

\begin{tabular}{lll}
\hline Tasks & $\begin{array}{l}\text { CHWs currently } \\
\text { performing task (\%), } \\
\mathbf{n = 2 8}\end{array}$ & $\begin{array}{l}\text { Clinicians reporting } \\
\text { task as shifted (\%), } \\
\mathbf{n = 1 9}\end{array}$ \\
\hline Register patients & $17(61 \%)$ & $12(63 \%)$ \\
Take vital signs & $18(64 \%)$ & $8(42 \%)$ \\
Dispense medications & $9(32 \%)$ & $5(26 \%)$ \\
Provide individual & $22(79 \%)$ & $8(42 \%)$ \\
education/counseling & & $16(84 \%)$ \\
Provide group education & $21(75 \%)$ & $7(37 \%)$ \\
Community mobilization & $18(64 \%)$ & $7(37 \%)$ \\
Linking health facility and & $10(36 \%)$ & $16(84 \%)$ \\
community & & \\
Defaulter tracing & $19(68 \%)$ & \\
\hline
\end{tabular}

and added-value services. CHWs worked an average of 5.3 hours/day (SD 2.5 hours) with $36 \%$ of their time spent on directly-shifted tasks and $64 \%$ on added-value work. On average, CHWs worked 12 of the 14 days surveyed (range 9 to 14 days). CHWs in rural areas and those based at dispensaries worked more hours per day, mostly due to a greater amount of time spent performing added-value tasks.

Table 5 displays the valuations of the CHW work recorded in the task diaries. The $17 \mathrm{CHWs}$ surveyed worked 1,139 hours valued at US\$ 999 (US\$ 0.88 per hour). Directly-shifted and added-value tasks accounted for nearly equal parts of this value: US\$ 459 (46\%) and US\$ 540 (54\%), respectively. The average value of each CHW's work per 14-day period was US\$ 59 (range US\$ 45 to 79). Averaged over a year, this represents a monthly salary of US\$ 117; if the average hourly CHW wage of US\$ 0.88 and a 40-hour workweek are used, the projected monthly salary is US\$ 147 .

\section{Modeling}

Clinician interviews revealed an average load of 35 patients per clinician per day (range 10 to 40). Using the model described (Figure 1 and Table 2) with the specified inputs, the average time for an individual patient clinic visit is 40 minutes, not including waiting time; thirty-three minutes of this time is accounted for by direct patient care and the remainder by general clinic services that indirectly benefit patients. The minimum amount of time for a clinic visit was 26 minutes and the maximum was 1 hour and 56 minutes.

Table 6 presents the work hours required by each $\mathrm{HCW}$ cadre to provide services to 35 patients per day in current and in idealized scenarios. This model projects that additional task shifting measures would free an estimated 6.1 hours per day of clinician time while adding 9.5 hours per day of $\mathrm{CHW}$ time, given that CHWs are less efficient at performing tasks than clinicians. If clinicians and CHWs were less efficient at performing tasks than assumed, it would have a greater effect on the potential time savings for clinicians than if they were more efficient.

Using this model, a health facility servicing 35 patients per day would require 0.5 clinician full-time equivalents (FTEs) and 2.4 CHW FTEs based on an 8-hour workday if the selected additional tasks were shifted. This is opposed to 1.3 clinician FTEs and 1.2 CHW FTEs required without further task shifting. The current scenario would cost US\$ 470 to 631 per month for wages depending on the type of facility and assuming a monthly CHW salary of US\$ 124 , which is a reasonable figure suggested from this study. The idealized scenario would cost an estimated US\$ 421 to 545 per month in wages, a 10.4 to $13.6 \%$ reduction in monthly wages for 
Table 4 Current community health worker (CHW) time contributions by facility type and population density

\begin{tabular}{|c|c|c|c|c|c|c|c|c|}
\hline & & \multirow{2}{*}{$\begin{array}{l}\text { Average number of daysworked } \\
\text { in } 14 \text { day period by } \mathrm{CHWs}\end{array}$} & \multicolumn{2}{|l|}{ Core Services } & \multicolumn{2}{|c|}{ Added value services } & \multicolumn{2}{|l|}{ All services } \\
\hline & & & $\begin{array}{l}\text { Avg hrs/day } \\
\text { (\% total) }\end{array}$ & Total hours & $\begin{array}{l}\text { Avg hrs/day } \\
\text { (\% total) }\end{array}$ & Total hours & Avg hrs/day & Total hours \\
\hline \multicolumn{9}{|l|}{ Facility type } \\
\hline Hospital & 11 & 12.1 & $2.2(42 \%)$ & 297 & $3.1(58 \%)$ & 418 & 5.4 & 715 \\
\hline Health center & 4 & 13.0 & $1.4(35 \%)$ & 74 & $2.6(65 \%)$ & 134 & 4.0 & 208 \\
\hline Dispensary & 2 & 14.0 & $1.3(16 \%)$ & 35 & $6.5(84 \%)$ & 181 & 7.7 & 216 \\
\hline \multicolumn{9}{|l|}{ Population density } \\
\hline Densely populated & 5 & 13.6 & $2.0(49 \%)$ & 135 & $2.1(51 \%)$ & 143 & 4.1 & 277 \\
\hline Mid-populated & 4 & 11.8 & $2.0(31 \%)$ & 93 & $4.4(69 \%)$ & 206 & 6.4 & 299 \\
\hline Sparely populated & 8 & 12.3 & $1.8(32 \%)$ & 179 & $3.9(68 \%)$ & 384 & 5.7 & 563 \\
\hline Total & 17 & 12.5 & 1.9 (36\%) & 406 & 3.4 (64\%) & 733 & 5.3 & 1139 \\
\hline
\end{tabular}

the same expected overall clinic productivity and would require fewer clinicians overall.

\section{Discussion}

Multiple studies and a recent meta-analysis support that lay HCWs can be as effective as higher skilled HCWs at providing a wide range of services [8-11,34]. In Kenya, CHWs providing care for antiretroviral drug delivery had similar outcomes of HIV control compared to standard clinic visits [12]. Our study's findings are consistent with these studies in that semistructured interviews and CHW task diaries demonstrated that facility-based CHWs are delivering a significant amount and variety of services, including tasks that have been directly-shifted from clinicians as well as those that are added-value to Kenya's health system. Tasks performed and the amount of task shifting varies among CHWs and as perceived by clinicians. It is important to note our study did not objectively measure quality of care provided by CHWs, however. In addition, we were unable to consider additional important aspects of the CHW workforce, including attrition, training and supervision.

A study in Rwanda on task shifting from physicians to nurses showed a $78 \%$ reduction in HIV-related physician workload as a result of implementing nurse-initiated antiretroviral therapy, saving up to 56 hours of physician time per month [35]. Our interviews revealed that task shifting to CHWs frees up 2.5 hours/day of clinicians' time on average. This is the first published data that we know of to quantify the time savings realized through CHW task shifting. In addition, our modeling exercise suggests transitioning other selected tasks from clinicians to CHWs could yield further significant time savings for clinicians while maintaining clinic productivity. These findings are consistent with studies showing costeffectiveness of CHW-based care [5-7,13,36,37].

Prior research has identified the lack of consistent and appropriate remuneration of $\mathrm{CHWs}$ as a significant barrier to the success of $\mathrm{CHW}$ programs. Paying CHWs appropriately for their work and providing clear delineation

Table 5 Monetary estimates of community health worker (CHW) contributions by facility type and population density for a 14-day study period and per month (all values in US\$)

\begin{tabular}{|c|c|c|c|c|c|c|c|c|}
\hline & \multirow[t]{2}{*}{ CHWs } & \multicolumn{2}{|c|}{ Directly shifted value } & \multicolumn{2}{|c|}{ Added value } & \multicolumn{3}{|c|}{ Total value } \\
\hline & & Total* & Per CHW* & Total & Per CHW & Total & Per CHW & Projected monthly value \\
\hline \multicolumn{9}{|l|}{ Facility type } \\
\hline Hospital & 11 & $\$ 357$ & $\$ 32$ & $\$ 311$ & $\$ 28$ & $\$ 668$ & $\$ 61$ & $\$ 121$ \\
\hline Health center & 4 & $\$ 80$ & $\$ 20$ & $\$ 100$ & $\$ 25$ & $\$ 180$ & $\$ 45$ & $\$ 90$ \\
\hline Dispensary & 2 & $\$ 26$ & $\$ 13$ & $\$ 135$ & $\$ 67$ & $\$ 160$ & $\$ 80$ & $\$ 160$ \\
\hline \multicolumn{9}{|l|}{ Population density } \\
\hline Densely populated & 5 & $\$ 149$ & $\$ 30$ & $\$ 106$ & $\$ 21$ & $\$ 255$ & $\$ 51$ & $\$ 102$ \\
\hline Mid-populated & 4 & $\$ 105$ & $\$ 26$ & $\$ 153$ & $\$ 38$ & $\$ 258$ & $\$ 64$ & $\$ 1$ \\
\hline Sparsely populated & 8 & $\$ 210$ & $\$ 26$ & $\$ 286$ & $\$ 36$ & $\$ 496$ & $\$ 62$ & $\$ 124$ \\
\hline Total & 17 & $\$ 463$ & $\$ 27$ & $\$ 545$ & $\$ 32$ & $\$ 1008$ & $\$ 59$ & $\$ 119$ \\
\hline
\end{tabular}

*Discounted for assumed time inefficiency of a CHW completing a task compared to a clinician. 
Table 6 Clinic model - effect of task shifting on health cadre work hours (based on 35 patient visits per day)

\begin{tabular}{|c|c|c|c|c|c|c|c|c|c|}
\hline \multirow[b]{2}{*}{ Health cadre } & \multicolumn{3}{|c|}{ Current scenario ${ }^{a}$ (hours per day) } & \multicolumn{3}{|c|}{ Ideal scenario $^{\text {b }}$ (hours per day) } & \multicolumn{3}{|c|}{ Difference $^{c}$ (hours per day) } \\
\hline & More efficient & Baseline & Less efficient & More efficient & Baseline & Less efficient & More efficient & Baseline & Less efficient \\
\hline Clinician & 4.7 & 10.2 & 19.8 & 1.8 & 4.1 & 8.2 & -2.9 & -6.1 & -11.6 \\
\hline $\mathrm{CHW}$ & 6.3 & 9.3 & 14.5 & 11.0 & 18.8 & 33.2 & +4.7 & +9.5 & +18.7 \\
\hline Total & 9.3 & 19.5 & 38.5 & 11.7 & 22.9 & 47.3 & +1.8 & +3.4 & +7.1 \\
\hline
\end{tabular}

This table presents work hours required by each healthcare worker to provide services to 35 patients per day in current and idealized scenarios. The amount of time required for each cadre to complete a service was varied by a factor of two, representing more and less efficiency, to determine the effects of these assumptions in the model. In the baseline current scenario, ${ }^{\mathrm{a}}$ clinicians work 10.2 hours per day while CHWs work 9.3 hours per day. In a baseline ideal scenario, ${ }^{\mathrm{b}}$ task shifting would result in clinicians working 4.1 hours per day while CHWs would work 18.8 hours per day. With appropriate tasks shifted to community health workers, 6.1 hours of clinician time would be freed, while 9.5 hours of community health worker time would be added. If healthcare workers were less efficient at performing tasks, this would have a greater effect on potential time savings than if they were more efficient. CHW, community health worker.

of CHW tasks has been shown to enhance CHW retention and improve sustainability of $\mathrm{CHW}$ programs. $[2,7,18,38-40]$ While a recent study enumerated health system-level costs associated with deploying CHWs using an estimate of current $\mathrm{CHW}$ wages [41], we attempted to quantify the monetary value of facility-based CHWs' work to provide concrete starting data points for the discussion of fair CHW compensation.

CHWs provided higher estimates of a fair monthly salary than clinicians (US\$ 178 and US\$ 128, respectively). Using the data from the CHW task diaries, we derived an average monthly valuation of current $\mathrm{CHW}$ services of US\$ 117, consistent with the clinicians' estimated $\mathrm{CHW}$ wage. While this calculation includes added value of CHWs it is likely an underestimate because we were not able to quantify important linkages that CHWs provide to health facilities.

Our modeling exercise suggests that further HCW skill optimization through additional task shifting and the expansion of CHW roles could justify employing 2.5 CHWs at an average-sized health facility at current workloads. Our clinic model is limited by the assumptions made regarding the average completion time for tasks; the proportion of patients in need of each service; the cadre that currently performs a task and which cadre would ideally do it; and the extra time required to complete a task after it has been shifted to a CHW. These assumptions affect the time required of each HCW to provide their services to an individual patient and the total daily cadre times. The sensitivity analysis showed significant variability in the projected clinician time savings and additional CHW time. This highlights the need for future research on these variables in resourcelimited clinics in order to better inform models.

Further, in Kenya, CHEWs are facility-based HCWs, but are meant to take on mostly a supervisory role of CHWs. As additional models of CHEWs and CHWs are developed, it may be necessary to create complementary cadres of CHWs: those that are predominately facilitybased and take on mostly task-shifted roles in the facility, and those that are community-based and engage mostly in added-value services that CHWs provide in the community.

There are several limitations to our estimates of the value of CHWs' work. Study subjects may have been more strongly supportive of $\mathrm{CHWs}$ given that all who were approached agreed to participate; this selection bias may have influenced results. Misclassification of tasks may also have occurred as they were categorized as directly-shifted or added-value based on semistructured interviews. The valuation of directly-shifted CHW tasks was affected by the uniform time adjustment made for the assumption that clinicians are more efficient than $\mathrm{CHWs}$; there is, however, likely significant variability in the relative efficiency of CHWs and clinicians. The research team chose the value of this discount factor based on input from clinicians and CHWs and their own experiences, not objective data due to an absence of relevant published data. The valuation of added-value tasks was based on a wage figure derived from our interviews with CHWs and clinicians. Because of this linkage, our valuation is less valid than if we had used an estimated CHW salary obtained by other means. CHWs had an incentive to provide a higher estimated $\mathrm{CHW}$ wage if they felt they stood to benefit from any future CHW compensation program, although CHWs may also have a better sense of what might be a fair living wage. Lastly, our estimate of clinician time liberated is limited since we did not simultaneously record clinicians' daily tasks. Nonetheless, we believe our estimates provide useful reference points for policy debates and future research.

In summary, CHWs are an important component of health service delivery in Kenya. They reduce the workload of clinicians, giving them more time to spend with patients, see additional patients and do higher-level job functions. They also provide services that would often not be provided otherwise and provide vital links between communities and health facilities. Our study attempts to put a value on these services to aid those involved with planning, financing, and implementing CHW programs in resource-limited settings. While compensation is just one component of the $\mathrm{CHW}$ programs 
that exist within complex parent health systems, it is important to appropriately address it upfront as these programs are designed, implemented, and scaled up.

\section{Conclusions}

CHWs are an important component of healthcare delivery in Kenya. The estimates herein provide starting points for fair compensation of CHWs, so they may be further integrated into health systems faced with a health worker shortage. Optimal utilization of CHWs will reduce the workload of strained health workers and improve access to health care.

\begin{abstract}
Abbreviations
CHEWs: community health extension workers; CHWs: community health workers; CS: community strategy; CU: community unit; FTE: full-time equivalents; HCWs: healthcare workers; MDGs: millennium development goals; NGOs: nongovernmental organizations.
\end{abstract}

\section{Competing interests}

The authors declare they have no competing interests.

\section{Authors' contributions}

LS contributed to the literature search, study design, data collection, data analysis, data interpretation and wrote the manuscript. DH contributed to the study design, data collection, data analysis, and data interpretation; generated the figure; and wrote the manuscript. MP contributed to study design, data analysis and data interpretation. JH conceived of the study design, contributed to data interpretation and wrote the manuscript. All authors read and approved the final manuscript.

\section{Acknowledgements}

This work was supported by the Center for Public Health Initiatives (CPHI) at the University of Pennsylvania. CPHI supported travel for the research team and transportation for health facility workers to participate in the study. CPHI did not contribute to study design or analysis.

\section{Author details}

'Johns Hopkins Bloomberg School of Public Health, 1000 E. Eager Street, Baltimore, MD 21202, USA. ${ }^{2}$ Baylor International Pediatrics AIDS Initiative, Maseru, Lesotho. ${ }^{3}$ University of Pennsylvania Wharton School, Philadelphia, PA, USA. ${ }^{4}$ Division of Infectious Diseases, University of Pennsylvania School of Medicine, Philadelphia, PA, USA.

Received: 8 September 2014 Accepted: 13 January 2015

Published: 30 January 2015

\section{References}

1. World Health Organization. Working together for health. In World health report 2006. Geneva: WHO; 2006. http://www.who.int/whr/2006/en/. Accessed 11 March 2011.

2. World Health Organization. Task shifting: rational redistribution of tasks among health workforce teams: global recommendations and guidelines. 2008. http://www.who.int/healthsystems/TTR-TaskShifting.pdf. Accessed 16 May 2011

3. Samb B, Celletti F, Holloway J, Van Damme W, De Cock KM, Dybul M. Rapid expansion of the health workforce in response to the HIV epidemic. N Engl J Med. 2007;357:2510-4.

4. Chen L, Evans T, Anand S, Boufford Jl, Brown H, Chowdhury M, et al. Human resources for health: overcoming the crisis. Lancet. 2004;364:1984-90.

5. Islam A, Wakai S, Ishikawa N, Chowdhury A, Vaughan J. Cost-effectiveness of community health workers in tuberculosis control in Bangladesh. Bull World Health Organ. 2002;80:445-50.

6. Nganda B, Wangombe J, Floyd K, Kangangi J. Cost and cost-effectiveness of increased community and primary care facility involvement in tuberculosis care in Machakos District Kenya. Int J Tuberc Lung Dis. 2003;7:S14-20.
7. Walker DG, Jan S. How do we determine whether community health workers are cost-effective? Some Core Methodological Issues. J Community Health. 2005;30:221-9.

8. Weidle PJ, Wamai N, Solberg P, Liechty C, Sendagala S, Were W, et al. Adherence to antiretroviral therapy in a home-based AIDS care programme in rural Uganda. Lancet. 2006;368:1587-94.

9. Morris MB, Chapula BT, Chi BH, Mwango A, Chi HF, Mwanza J, et al. Use of task-shifting to rapidly scale-up HIV treatment services: experiences from Lusaka, Zambia. BMC Health Serv Res. 2009;9:1-9.

10. Callaghan M, Ford N, Schneider H. A systematic review of task-shifting for HIV treatment and care in Africa. Hum Resour Health. 2010;8:1-9.

11. Bemelmans $M$, van den Akker T, Ford N, Philips M, Zachariah R, Harries A, et al. Providing universal access to antiretroviral therapy in Thyolo, Malawi through task shifting and decentralization of HIV/AIDS care. Trop Med Int Heal. 2010;15:1413-20.

12. Selke HM, Kimaiyo S, Sidle JE, Vedanthan R, Tierney WM, Shen C, et al. Taskshifting of antiretroviral delivery from health care workers to persons living with HIV/AIDS: clinical outcomes of a community-based program in Kenya. J Acquir Immune Defic Syndr. 2010;55:483-90.

13. Datiko $D$, Lindtjorn B. Cost and cost-effectiveness of smear-positive tuberculosis treatment by health extension workers in southern Ethiopia: a community randomized trial. PLoS One. 2010;5:e9158.

14. Mwai GW, Mburu G, Torpey K, Frost P, Ford N, Seeley J. Role and outcomes of community health workers in HIV care in sub-Saharan Africa: a systematic review. J Int AIDS Soc. 2013;16:1-14.

15. Perry H, Zulliger R. How effective are community health workers? 2012. http:// www.coregroup.org/storage/Program_Learning/Community_Health_Workers/ review\%20of\%20chw\%20effectiveness\%20for\%20mdgs-sept2012.pdf. Accessed 26 January 2014.

16. Republic of Kenya Ministry of Public Health and Sanitation. Report of the review of the implementation of the community health strategy. Kenya: Nairobi; 2009.

17. Partnership The United National Development Program Japan and GROOTS Kenya. Compensation for contribution report: a baseline study. Kenya: Nairobi; 2009.

18. Lehmann U, Sanders D. Community health workers: What do we know about them? World Health Organization: Geneva, Switzerland; 2007. http://www.who.int/hrh/documents/community_health_workers.pdf. Accessed 16 May 2011.

19. Hermann K, Van Damme W, Pariyo GW, Schouten E, Assefa Y, Cirera A, et al. Community health workers for ART in sub-Saharan Africa: learning from experience-capitalizing on new opportunities. Hum Resour Health. 2009;7:31.

20. Jaskiewicz W, Tulenko K. Increasing community health worker productivity and effectiveness: a review of the influence of the work environment. Hum Resour Health. 2012;10:38.

21. Republic of Kenya Ministry of Health. Taking the Kenya Essential Package for Health to the Community: A Strategy for the Delivery of Level One Services. Nairobi, Kenya: Republic of Kenya Ministry of Health; 2006. http://www. communityledtotalsanitation.org/sites/communityledtotalsanitation.org/files/ community_strategy.pdf. Accessed 17 May 2011.

22. Mireku M, Kiruki M, McCollum R, Taegtmeyer M, de Koning K, Otiso L. Context Analysis: close-to-community health services providers in Kenya. 2014 http://www.reachoutconsortium.org/media/1837/kenyacontextanalysis jul2014compressed.pdf. Accessed 30 November 2014.

23. Republic of Kenya Ministry of Public Health and Sanitation. 8 March 2011). Policy shift on community strategy (Ref. No: MPHS/ADM/2/30 Vol.IV. Nairobi, Kenya; 2011.

24. Government of Kenya. Revised scheme of service for clinical officers Nairobi, Kenya: Government of Kenya; 2010. http://clinicalofficerscouncil.org/ resources/SCHEME_OF_SERVICES_FOR_CLINICAL_OFFICERS.pdf. Accessed 30 November 2014

25. Mbindyo P, Blaauw D, English M. The role of Clinical Officers in the Kenyan health system: a question of perspective. Hum Resour Health. 2013;11:32.

26. Nursing Council of Kenya. Education Information: Approved Nursing Programmes. Nairobi County, Kenya; 2014. http://nckenya.com/edu/ programmes/. Accessed 30 November 2014

27. Chankova S, Muchiri S, Kombe G. Health workforce attrition in the public sector in Kenya: a look at the reasons. Hum Resour Health. 2009;7:58

28. World Health Organization. Global Atlas of the Health Workforce. Geneva, Switzerland: World Health Organization; 2010. http://apps.who.int/ globalatlas/default.asp. Accessed 13 April 2011. 
29. One Million Community Health Workers Campaign. Update from the field: Kenyan community health services works to establish national standards. New York, New York; 2014. http://1 millionhealthworkers.org/2014/08/25/ update-from-the-field-kenyan-community-health-services-works-to-establishnational-standards/. Accessed 30 November 2014.

30. Government of Kenya and UNICEF. Evaluation report of the community health strategy implementation in Kenya. Nairobi, Kenya: Government of Kenya and UNICEF; 2010. http://www.unicef.org/evaldatabase/files/ 14_2010_HE_002_Community_Strategy_Evaluation_report_October_2010. pdf. Accessed 30 November 2014

31. Greene JC, Caracelli VJ, Graham WF. Toward a conceptual framework for mixed-method evaluation designs. Educ Eval Policy Anal. 1989;11:255-74.

32. Columbia University Center for International Earth Science Information Network. Gridded population of the world. New York, New York; 2013. http://sedac.ciesin.columbia.edu/gpw/country.jsp?iso=KEN. Accessed 14 May 2011.

33. Kenya National Bureau of Statistics. Country stats. Nairobi, Kenya; 2015. http://www.knbs.or.ke/. Accessed 14 May 2011.

34. Lassi ZS, Gometto G, Huicho L, Bhutta ZA. Quality of care provided by mid-level health workers: systematic review and meta-analysis. Bull World Health Organ. 2013;91:824-33. Doi:10.2471/BLT.13.118786.

35. International AIDS Society, Chung J, O'Brien M, Price J, Shumbusho F. Quantification of physician-time saved in a task shifting pilot program in Rwanda. AIDS 2008 - XVII Int. Geneva, Switzerland: AIDS Conference. www.aids2008.org/Pag/Abstracts.aspx?SID=255\&AID=6043. Accessed 16 May 2011.

36. Lewin S, Munabi-Babigumira S, Glenton C, Daniels K, Bosch-Capblanch X, van $W y k B E$, et al. Lay health workers in primary and community health care for maternal and child health and the management of infectious diseases. Cochrane Database Syst Rev. 2010;28:1-208.

37. Hongoro C, McPake B. How to bridge the gap in human resources for health. Lancet. 2004;364:1451-6.

38. Schneider $\mathrm{H}, \mathrm{Hlophe} H$, van Rensburg D. Community health workers and the response to HIV/AIDS in South Africa: tensions and prospects. Health Policy Plan. 2008;23:179-87.

39. Philips $M$, Zachariah $R$, Venis $S$. Task shifting for antiretroviral treatment delivery in sub-Sahran Africa: not a panacea. Lancet. 2008;371:682-4.

40. Nkonki L, Cliff J, Sanders D. Lay health worker attrition: important but often ignored. Bull World Health Organ. 2011;89:919-23.

41. McCord GC, Liu A, Singh P. Deployment of community health workers across rural sub-Saharan Africa: financial considerations and operational assumptions. Bull World Health Organ. 2013;91:244-53B.

doi:10.1186/1478-4491-13-6

Cite this article as: Sander et al:: Time savings - realized and potential and fair compensation for community health workers in Kenyan health facilities: a mixed-methods approach. Human Resources for Health 2015 13:6.

\section{Submit your next manuscript to BioMed Central and take full advantage of:}

- Convenient online submission

- Thorough peer review

- No space constraints or color figure charges

- Immediate publication on acceptance

- Inclusion in PubMed, CAS, Scopus and Google Scholar

- Research which is freely available for redistribution 